\title{
Correction to: Canard Cycles
}

\section{Correction to: \\ P. De Maesschalck et al., \\ Canard Cycles, Ergebnisse der Mathematik und ihrer \\ Grenzgebiete. 3. Folge/A Series of Modern Surveys in \\ Mathematics 73, https://doi.org/10.1007/978-3-030-79233-6}

Due to an unfortunate oversight, background TeX indexing commands were included in the text of chapters 1,2 and 4. This has now been corrected.

The updated online versions of the chapters can be found at https://doi.org/10.1007/978-3-030-79233-6_1

https://doi.org/10.1007/978-3-030-79233-6_2

https://doi.org/10.1007/978-3-030-79233-6_4

(C) The Author(s), under exclusive license to Springer Nature Switzerland AG 2022 